\title{
The need for branching fraction measurements in multiply-charged ions
}

\author{
L J Curtis, S R Federman, S Torok, M Brown, S Cheng, R E Irving \\ and $\mathrm{R} M$ Schectman
}

Department of Physics and Astronomy, University of Toledo, OH 43606, USA

E-mail: steven.federman@utoledo.edu

Received 25 September 2006

Accepted for publication 9 March 2007

Published 11 May 2007

Online at stacks.iop.org/PhysScr/75/C1

\begin{abstract}
Much progress has been made in the measurement of oscillator strengths in neutral and singly-ionized atoms, providing a database for use in important applications. However, for multiply-charged ions, measured data for oscillator strengths are almost exclusively limited to low-lying unbranched transitions. Although extensive measurements of ionic lifetimes exist, the lack of branching fraction measurements in multiply-charged ions prevents these data from being converted to oscillator strengths. A significant factor leading to this deficiency involves the lack of adequate line intensity calibration standards in the vacuum ultraviolet spectral region 2000-400 $\AA$. Here we review the interrelationships connecting these rate parameters, indicate some of the important applications for which they are needed, describe the experimental limitations that currently exist, and suggest possible methods for extending these measurements below $1000 \AA$.
\end{abstract}

PACS numbers: 31.15.Ct, 32.30.-r, 32.30.Jc, 32.70.Fw, 39.90.+d, 32.70.Cs

\section{Introduction}

Many important applications of atomic data require a knowledge of emission and absorption transition rate parameters in atomic ions. These quantities are required, e.g. to deduce elemental abundances from astrophysical spectra, to determine impurity concentrations from fusion plasma spectra, to interpret data from fast ion beam and heavy ion storage ring measurements, to test theoretical methods through transition-by-transition comparisons with experiment, and to obtain precise semiempirical interpolations and extrapolations of measured data along isoelectronic sequences (ions that have the same number of electrons, but a sequentially increasing nuclear charge).

The experimental determination of these quantities [1] can be achieved either through the use of absolute emission, absorption, and dispersion measurements, or through intensity-calibrated measurements of relative intensities combined with time-resolved measurements of upper level lifetimes. Methods now exist whereby measurements of lifetimes can be carried out for levels in nearly any ionization stage of any atom, and relative intensity measurements have been made for neutral atoms as well as singly- and some doublycharged ions. However, relative intensity measurements in multiply-charged ions require calibration in the vacuum ultraviolet (VUV) spectral region (2000-400 ̊). These measurements are challenging, and few data currently exist.

In this paper, we review the interrelationships connecting these rate parameters, indicate some of the important applications for which they are needed, describe the experimental limitations that currently exist, and suggest possible methods for extending these measurements.

\section{Definitions of emission and absorption rate parameters}

This section presents a short review of the relationships among quantities that prescribe the emission and absorption of atomic radiation. A comprehensive exposition of these concepts, terminology, and notation can be found in [2].

A spectral absorption or emission line arising from a transition between an upper level $\mathrm{u}$ and a lower level 1 in a free atom is characterized by its wavelength $\lambda_{\mathrm{ul}}$, its transition rate, and its shape. For emission, the transition rate per atom is specified by the 'transition probability' $A_{\mathrm{ul}}$ (a misnomer, since it denotes a rate and not a probability). For absorption, the transition rate is specified by the oscillator strength $f_{\text {lu }}$ (a dimensionless quantity defined relative to the classical 
harmonic oscillator rate). In emission the shape is prescribed by the natural Lorentzian line width $\Gamma_{\mathrm{ul}}=\hbar / \tau_{\mathrm{u}}+\hbar / \tau_{1}$, where $\tau_{\mathrm{u}}$ and $\tau_{1}$ are the lifetimes (the exponential mean lives) of the levels. In absorption the shape also involves the degree of saturation of the removal of the incident radiation.

For an ensemble of atoms in emission, the radiated intensity $I_{\mathrm{ul}}$ (in photons/sec) is given by

$$
I_{\mathrm{ul}}=N_{\mathrm{u}} A_{\mathrm{ul}}
$$

where $N_{\mathrm{u}}$ is the instantaneous population of the upper level. For absorption of continuum radiation by a sample of atoms, the equivalent width $W_{\text {lu }}$ (a measure of the fractional absorption that corrects for saturation effects) is given by the proportionality

$$
W_{\text {lu }} \propto N_{1} L f_{\text {lu }}
$$

where $N_{1}$ is the population density of the lower level and $L$ is the path length of the radiation through the sample. Thus, to determine sample populations (and thereby elemental abundances, excitation functions, etc.) from emission and absorption measurements, knowledge of the quantities $A_{\mathrm{ul}}$ and $f_{\text {lu }}$ is required.

Both the absorption and emission processes involve the same quantum mechanical line strength factor $S_{\mathrm{ul}}$ [3], given by

$$
S_{\mathrm{ul}}=S_{\mathrm{lu}}=\left|\left\langle\Psi_{1}\left|\mathbf{r} / a_{0}\right| \Psi_{\mathrm{u}}\right\rangle\right|^{2}
$$

where $a_{0}=0.529 \AA$ is the Bohr radius. This theoretically specifies both the transition probability and the oscillator strength through the relationships

$$
g_{\mathrm{u}} A_{\mathrm{ul}}\left(\mathrm{ns}^{-1}\right)=\left[\frac{1265.38}{\lambda_{\mathrm{ul}}(\AA)}\right]^{3} S_{\mathrm{ul}}
$$

and

$$
g_{1} f_{\mathrm{lu}}=\left[\frac{303.75}{\lambda_{\mathrm{ul}}(\AA)}\right] S_{\mathrm{ul}},
$$

where $g_{\mathrm{u}}$ and $g_{1}$ are the statistical weights (the degeneracies in magnetic quantum number) of the upper and lower levels. Combining equations (4) and (5), the absorption and emission rates are related to each other by

$$
g_{1} f_{\mathrm{lu}}=\left[\frac{\lambda_{\mathrm{ul}}(\AA)}{2582.68}\right]^{2} g_{\mathrm{u}} A_{\mathrm{ul}}\left(\mathrm{ns}^{-1}\right),
$$

hence measurements of rate constants made in emission can be used to interpret data obtained in absorption and vice versa.

If the upper level has decay channels to more than one lower level, the decay is said to be 'branched'. The ratio of intensities of radiation emitted in transitions from the same upper level $\mathrm{u}$ to lower levels 1 and $\mathrm{l}^{\prime}$ is called the branching ratio, and is given by $A_{\mathrm{ul}} / A_{\mathrm{ul}^{\prime}}$. The total diminution of the population of the upper level is given by the sum of the rates of all of the decay channels, hence the reciprocal lifetime is given by

$$
1 / \tau_{\mathrm{u}}=\sum_{1} A_{\mathrm{ul}}
$$

Thus the decay curves measured in all branches from the same upper level will exhibit the same time dependence, but each will have a characteristic overall intensity.
The ratio of the intensity in a specific branch to the sum of all intensities from the same upper level is the branching fraction $B_{\mathrm{ul}}$, given by

$$
B_{\mathrm{ul}}=A_{\mathrm{ul}} / \sum_{l^{\prime}} A_{\mathrm{ul}^{\prime}}=\tau_{\mathrm{u}} A_{\mathrm{ul}} .
$$

Since transitions from the same upper level involve the same population and the same lifetime, the emitted intensity ratio is given by the branching ratio $I_{\mathrm{ul}} / I_{\mathrm{ul}^{\prime}}=B_{\mathrm{ul}} / B_{\mathrm{ul}^{\prime}}$. The detected intensities will differ from the emitted intensities by factors of the detection efficiency, which must be determined as a function of wavelength using a calibration standard.

Lifetime determinations involve measuring the intensity at a given wavelength at various times, so they require only a calibration of the time base. Relative intensity measurements of the individual decay channels involve measuring the intensity at various wavelengths at the same time, thus requiring a more difficult calibration of the detection efficiency as a function of wavelength. This important issue is discussed further below.

\section{Applications}

There are many applications that require a knowledge of transition probabilities and oscillator strengths in ions. A few examples are described briefly here.

In astrophysics, satellite-borne observatories such as Solar and Heliospheric Observatory (SOHO) and Far Ultraviolet Spectroscopic Explorer (FUSE) are producing much data in the VUV. SOHO is providing plasma diagnostics of ionized atoms in the solar atmosphere, chromosphere, and corona, and FUSE is exploring the interstellar medium and remnants of supernova explosions using spectra of, e.g. O VI.

In the development of controlled nuclear fusion devices, impurity transport studies utilizing Tokamak Experiment for Technology Oriented Research (TEXTOR) follow the time variation of highly ionized species of injected impurities through their spectra.

The use of fast ion beams to produce highly ionized atoms by acceleration and subsequent stripping of bound electrons by solid or gaseous targets has provided a wealth of timeresolved spectroscopic data. The limitations of single-pass arrangements (which restrict these experiments to systems with a short time interval between production and destruction) have been removed by the development of heavy-ion storage rings, producing much new lifetime data. To convert these lifetimes to transition probabilities requires measurements of branching fractions.

While sophisticated theoretical methods now permit reliable calculations for atoms and ions up through the iron group, the precision decreases substantially as the number and complexity of electron configurations increases. Significant discrepancies exist between theoretical calculations and experimental measurements of lifetimes, the origins of which are difficult to analyse when the transition rates for the individual branches are unknown.

Because of the complexity of heavy, many-electron ions, semiempirical parametrizations of measured data often yield interpolative and extrapolative predictions of much higher 
accuracies than $a b$ initio theoretical calculations. A powerful empirical tool involves the study of isoelectronic sequences. Isoelectronic expositions of line strength data (deduced from measured transition probabilities using equation (4)) are found to exhibit slowly varying, nearly linear variations as a function of reciprocal screened charge [3]. These expositions have predictive capabilities of high accuracy. Unfortunately, because of the lack of branching fraction data for highly ionized atoms, these methods have been applied primarily to low-lying unbranched transitions. Examples [4] include the $n \mathrm{~s} \quad{ }^{2} \mathrm{~S}_{1 / 2}-n \mathrm{p} \quad{ }^{2} \mathrm{P}_{1 / 2,3 / 2}$ transitions in the $\mathrm{Li}(n=2)$, $\mathrm{Na}(n=3), \mathrm{Cu}(n=4)$, and $\mathrm{Ag}(n=5)$ sequences, the $n \mathrm{~s}^{2}{ }^{1} \mathrm{~S}_{0}-n \mathrm{~s} n \mathrm{p}{ }^{1,3} \mathrm{P}_{1}$ transitions in the $\mathrm{Be}(n=2), \mathrm{Mg}(n=3)$, $\mathrm{Zn}(n=4), \mathrm{Cd}(n=5)$ and $\mathrm{Hg}(n=6)$ sequences, and the $n \mathrm{p}^{6}{ }^{1} \mathrm{~S}_{0}-n \mathrm{p}^{5}(n+1) \mathrm{s}{ }^{1,3} \mathrm{P}_{1}$ transitions in the $\mathrm{Ne}(n=2)$ and $\operatorname{Ar}(n=3)$ sequences.

\section{Intensity calibration methods}

For neutral atoms, the spectral lines tend to lie in the visible and near UV spectral region, for which reliable line intensity standards exist [5-8]. An extensive database has been produced by combining lifetime measurements with relative intensity measurements obtained using calibrated grating spectrometers.

For ionized atoms, the spectral lines tend to fall in the VUV region. Experimentally, this portion of the electromagnetic spectrum can be subdivided into the longer VUV region $2000-1050 \AA$ (2000 $\AA$ being the cutoff due to the opacity of air, $1050 \AA$ being the cutoff for solid window materials) and the extreme UV (EUV) extending from the windowless VUV down to the soft x-ray region.

In the long VUV region continuum sources can be used, such as wall-stabilized arcs (e.g. [9]), calibrated deuterium lamps (e.g. [10]), and synchrotron radiation (e.g. [11]). In the EUV, line calibration standards have also been developed using hollow cathode [12] and Penning ionization [13] methods.

Where applicable, the recent development of VUV Fourier transform spectrometers (FTS) $[11,14]$ offers distinct advantages for the measurement of branching fractions. This is due to the broadband transmittance of the Michelson interferometer used, as compared to the strong variability in reflective efficiency of a blazed grating spectrometer. Thus FTS methods are providing an increasing database in singly (and, in a few cases, doubly) ionized atoms.

However, for higher stages of ionization, fast ion beam excitation methods provide the most widely applicable method. Calibration of radiation emitted in-flight by a particle beam requires different considerations than calibration of radiation emitted by a lab-fixed thermal discharge. The use of fast ion beams introduces Doppler broadenings and shifts, polarizations due to anisotropic excitation, etc. While the low density of the beam provides a field-free collision-free source, it also leads to low light levels. If a continuum source of known spectral emission is used to calibrate a system designed to detect low intensity line spectra, there can be an intensity mismatch. For these measurements in-beam standards have significant advantages over lab-fixed standards.
One solution to this problem is to use a two-step process whereby a lab-fixed continuum calibration source is used to measure relative branching ratios in the line spectrum of a conveniently chosen ion. This ion can then be accelerated to a velocity that is matched to that of an ion to be studied. In this manner an in-beam intensity calibration could be performed with Doppler effects matched to that of the ion studied.

Another possibility is to identify types of transition arrays for which the relative intensities can be reliably predicted, either by theoretical or by semiempirical methods. We now explore this possibility in more detail.

\section{Development of intensity standards using measured intermediate coupling (IC) relationships}

Theoretical models for the study of multielectron atoms are usually built using basis sets drawn from solutions obtained for simple atoms. The radial part of the wavefunction is constructed from a 'complex' of electron configurations labelled by the hydrogenic quantum numbers $n$ and $\ell$ which possess the same parity. If there is a need to include two or more of these configurations, the system is said to possess 'configuration interaction' (CI). If a single configuration is adequate to describe the system experimentally, CI is said to be absent or negligible. Similarly, the angular part of the wavefunction is constructed from a set of $L S$-coupled terms (or, alternatively, $j j$-coupled terms) which all possess the same total angular momentum $J$. If it is necessary to include two or more of these $L S$ basis vectors to describe the system experimentally, it is said to possess IC. The manifold of transitions that connect two configurations is called a 'transition array, and the relative intensities of these lines can be predicted from the IC amplitudes that characterize the system.

Thus, a promising method for establishing relative intensity calibration standards involves the study of transitions between two complexes each of which is adequately described by a single configuration. If neither complex is significantly affected by CI, but one or both possess IC, the amplitudes of the $L S$ components specify the deviation from $L S$ values of both the energy levels and the transition rates. If there is no significant branching of the upper configuration to other complexes, then precise empirical predictions of branching fractions can be made using IC amplitudes deduced from measured energy level spacings.

While this approach utilizes certain assumptions concerning the theoretical model, the predictions are semiempirical rather than theoretical. They are based on experimental measurements, and thus have the potential to be more accurate than $a b$ initio theoretical predictions, particularly for complex many-electron systems.

In the nonrelativistic approximation for an assumed central potential, the theoretical specification of a transition array between two pure configurations separates into the product of a radial portion that is common to the entire array, and an angular portion that is specific to each line. Thus the radial transition element is, at least in this approximation, the same for all members of the array. If perturbations due to the direct and exchange interactions of the electrons (Slater parameters) and the magnetic coupling between the spin of 
each electron and its orbital motion relative to the nucleus (spin-own-orbit coupling) are included, angular momentum admixtures are formed connecting unperturbed $L S$-coupled basis vectors possessing the same total angular momentum $J$. The IC amplitudes characterize these couplings, and thereby specify both the energy level splittings and the branching fractions. Under these assumptions the radial transition moment cancels when branching ratios are computed, and the branching fractions can be computed by combining $L S$ coupling values with empirical IC amplitudes. Since the number of measured energy levels usually exceeds the number of IC amplitudes needed to describe them, the system is overdetermined and the validity of the single configuration model can be tested.

The factorization of the angular and radial wavefunctions allows the line strength factor of equation (4) to be written as

$$
S_{\mathrm{ul}} \rightarrow \mathcal{L}_{\mathrm{ul}}^{2}|\langle u|r| l\rangle|^{2},
$$

where $\mathcal{L}_{\mathrm{ul}}$ is a quantity involving $L S$ coupling transition matrices and empirical IC amplitudes and is specific to each line. The radial transition element $\langle u|r| l\rangle$ is independent of angular quantities, and is the same for the entire transition array. In this case the ratio of branching fractions for any pair of lines in the array is obtained by combining equations (4), (8) and (9) to obtain

$$
\frac{B_{\mathrm{ul}}}{B_{\mathrm{u}^{\prime} \mathrm{l}^{\prime}}}=\frac{\tau_{\mathrm{u}} \mathcal{L}_{\mathrm{ul}}^{2}}{\tau_{\mathrm{u}^{\prime}} \mathcal{L}_{\mathrm{u}^{\prime} \mathrm{l}^{\prime}}^{2}} \frac{g_{\mathrm{u}^{\prime}} \lambda_{\mathrm{u}^{\prime} 1^{\prime}}^{3}}{g_{\mathrm{u}} \lambda_{\mathrm{ul}}^{3}} .
$$

The application of these approximations is simplest for the comparison of relative intensities of branched decays from the same upper level to various lower level decay channels. In this case the intensities involve the same upper level population and lifetime, and equation (10) becomes the branching fraction

$$
\frac{B_{\mathrm{ul}}}{B_{\mathrm{ul} l^{\prime}}}=\frac{\mathcal{L}_{\mathrm{ul}}^{2} \lambda_{\mathrm{ul}^{\prime}}^{3}}{\mathcal{L}_{\mathrm{ul}^{\prime}}^{2} \lambda_{\mathrm{ul}}^{3}}
$$

One way to test the assumption that the radial matrix element is the same for the entire multiplet is to measure the lifetimes of the upper levels for the various $J$-dependent fine structure levels. With these assumptions, the lifetimes will not be identical, because of the dependence on observed wavelengths and empirical IC coefficients. From equations (4) and (7), the ratio of the lifetimes of the upper levels $u$ and $u^{\prime}$ is given by

$$
\frac{\tau_{\mathrm{u}}}{\tau_{\mathrm{u}^{\prime}}}=\frac{g_{\mathrm{u}}}{g_{\mathrm{u}^{\prime}}} \frac{\sum_{\mathrm{l}^{\prime}} \mathcal{L}_{\mathrm{u}^{\prime} l^{\prime}}^{2} / \lambda_{\mathrm{u}^{\prime} l^{\prime}}^{3}}{\sum_{\mathrm{l}} \mathcal{L}_{\mathrm{ul}}^{2} / \lambda_{\mathrm{ul}}^{3}} .
$$

Predicted values can be compared with ratios of measured lifetimes to test the assumptions of the model.

\subsection{Test of reliability: the neutral Si, Ge, and Sn atoms}

A test of this approach to the $n \mathrm{~s}^{2} n \mathrm{p}^{2}-n \mathrm{~s}^{2} n \mathrm{p}(n+1) \mathrm{s}$ transitions in the neutral atoms Si I $(n=3)$, Ge I $(n=4)$ and Sn I $(n=5)$ has been carried out [15-17]. For these neutral atoms the transitions lie in the air wavelength region (longward of $2000 \AA$ ). Thus intensity calibration standards are available [5-8], and precision branching fraction measurements have been made [18-20].

For this system there are seven energy level splittings (among the ${ }^{3} \mathrm{P}_{0,1,2},{ }^{1} \mathrm{D}_{2},{ }^{1} \mathrm{~S}_{0}$ levels in the ground configuration and among the ${ }^{3} \mathrm{P}_{0,1,2}^{\mathrm{o}},{ }^{1} \mathrm{P}_{1}^{\mathrm{o}}$ levels in the excited configuration) that are described by four Slater and spin-orbit parameters $\left(F_{2}, \zeta_{\mathrm{pp}}\right.$ and $\left.G_{1}, \zeta_{\mathrm{p}}\right)$ [3]. These parameters were deduced from the energy splittings, and their overdetermination was used to test the single configuration assumption. The four fitted parameters accurately reproduced the seven measured splittings, providing confidence that both the lower and upper manifolds are effectively pure configurations. Since there are, for these configurations, at most two levels that share the same value of $J$, each normalized pair of IC amplitudes can be economically expressed as a single singlet-triplet mixing angle. The fitted Slater and spin-orbit parameters specified the singlet-triplet mixing angles, which were used to compute the branching fractions (simple algebraic expressions for the angular factors $\mathcal{L}_{\mathrm{ul}}$ for these levels are available [3,16]). The results are displayed in table 1 . Here, and in subsequent tables, primes are affixed to $L S$ spectroscopic term symbols affected by singlet-triplet mixing to indicate that the notation is nominal.

As can be seen from this tabulation, the semiempirical calculations generally agree to within quoted uncertainties with the measured values. Table 1 also lists the pure $L S$ values. These are fairly close to the semiempirical values for the spin-allowed transition, indicating that $\mathrm{CI}$ is relatively small. For the spin-forbidden transitions, CI causes the branching to deviate from zero.

The fact that these seven energy spacings are accurately characterized by four Slater and spin-orbit parameters indicates that effects of $\mathrm{CI}$ are very small. The fact that the spin-allowed branching fractions deviate only slightly from the $L S$ values indicates that IC is a small correction. Thus, if the IC corrections are only a few percent, and these corrections are themselves accurate to within a few percent, it is reasonable to conclude that these semiempirical branching fractions are accurate to within a percent. An interesting additional question then concerns the spinforbidden branching fractions which, being zero for pure $L S$ coupling, are given entirely by the IC corrections. Since the semiempirical branching fractions are deduced from measured energy level data, it is conceivable that the empirical spin-forbidden branching fractions are more reliable than the corresponding values obtained from $a b$ initio wavefunctions, since the energy levels predicted usually differ slightly from experiment.

\subsection{Lifetime and branching fraction measurements in P II}

In order to investigate whether these semiempirical calculations are also reliable for spin-allowed transitions in ions, and to examine the agreement between semiempirical and $a b$ initio calculations for spin-forbidden transitions, a study has been made of P II in the Si sequence [21]. A comparison between semiempirical estimates, $a b$ initio calculations, and measured values for branching fractions is presented in table 2. The agreement among determinations is quite striking. 
Table I. Semiempirical (SE) and measured (M) branching fractions for $n \mathrm{~s}^{2} n \mathrm{p}^{2}-n \mathrm{~s}^{2} n \mathrm{p}(n+1) \mathrm{s}$ transitions in neutral atoms. The energy level database is tabulated in [15]. Parentheses following the measured values indicate quoted uncertainties in the last figure. $L S$-coupling values (both decimal and rational fraction) are provided to characterize the IC corrections.

\begin{tabular}{|c|c|c|c|c|c|c|c|}
\hline \multirow[b]{3}{*}{ Transition } & \multicolumn{6}{|c|}{ Branching fractions (\%) } & \multirow[b]{3}{*}{$L S$} \\
\hline & \multicolumn{2}{|r|}{ Si I } & \multicolumn{2}{|c|}{ Ge I } & \multicolumn{2}{|c|}{ Sn I } & \\
\hline & SE & $\mathrm{M}^{\mathrm{a}}$ & $\mathrm{SE}$ & $\mathrm{M}^{\mathrm{b}}$ & SE & $\mathbf{M}^{\mathrm{c}}$ & \\
\hline${ }^{3} \mathrm{P}_{0}^{\prime} \leftarrow{ }^{3} \mathrm{P}_{1}^{\mathrm{o}^{\prime}}$ & 33.3 & $33.3(3)$ & 31.2 & $32.5(16)$ & 32.3 & 27 & $33.3\left(\frac{1}{3}\right)$ \\
\hline${ }^{3} \mathrm{P}_{1} \leftarrow$ & 24.7 & $24.7(4)$ & 21.2 & $22.1(11)$ & 17.5 & 17 & $25.0\left(\frac{1}{4}\right)$ \\
\hline${ }^{3} \mathrm{P}_{2}^{\prime} \leftarrow$ & 41.1 & $40.7(4)$ & 38.1 & 37.1(19) & 39.7 & 39 & $41.7\left(\frac{5}{12}\right)$ \\
\hline${ }^{1} \mathrm{D}_{2}^{\prime} \leftarrow$ & 0.88 & $1.2(1)$ & 8.8 & $8.1(8)$ & 10.0 & 17 & 0 \\
\hline${ }^{1} S_{0}^{\prime} \leftarrow$ & 0.06 & $<0.20(6)$ & 0.52 & $0.23(2)$ & 0.5 & - & 0 \\
\hline${ }^{3} \mathrm{P}_{1} \leftarrow{ }^{3} \mathrm{P}_{2}^{\mathrm{o}}$ & 25.2 & $24.6(3)$ & 26.4 & $27.2(14)$ & 28.3 & 22 & $25.0\left(\frac{1}{4}\right)$ \\
\hline${ }^{3} \mathrm{P}_{2}^{\prime} \leftarrow$ & 74.8 & $75.4(3)$ & 73.1 & $72.1(14)$ & 68.5 & 71 & $75.0\left(\frac{3}{4}\right)$ \\
\hline${ }^{1} \mathrm{D}_{2}^{\prime} \leftarrow$ & 0.02 & $0.027(4)$ & 0.53 & $0.72(7)$ & 3.2 & 7 & 0 \\
\hline${ }^{3} \mathrm{P}_{0}^{\prime} \leftarrow{ }^{1} \mathrm{P}_{1}^{\mathrm{o}^{\prime}}$ & 0.24 & $0.30(2)$ & 2.9 & $4.6(5)$ & 4.2 & 8 & 0 \\
\hline${ }^{3} \mathrm{P}_{1} \leftarrow$ & 0.25 & $0.20(2)$ & 3.3 & $3.6(4)$ & 6.8 & 4 & 0 \\
\hline${ }^{3} \mathrm{P}_{2}^{\prime} \leftarrow$ & 0.15 & $0.20(2)$ & 1.0 & $1.68(17)$ & 0.01 & - & 0 \\
\hline${ }^{1} \mathrm{D}_{2}^{\prime} \leftarrow$ & 92.0 & $93.4(2)$ & 86.2 & $86.1(14)$ & 82.2 & 88 & $83.3\left(\frac{5}{6}\right)$ \\
\hline${ }^{1} \mathrm{~S}_{0}^{\prime} \leftarrow$ & 7.4 & $5.70(12)$ & 6.6 & $4.0(4)$ & 6.8 & - & $16.7\left(\frac{1}{6}\right)$ \\
\hline
\end{tabular}

${ }^{\mathrm{a}}$ Smith et al [18]. ${ }^{\mathrm{b}}$ Li et al [19]. ${ }^{\mathrm{c}}$ Lotrian et al [20].

Table 2. Semiempirical, theoretical and measured branching fractions for P II. The energy level database is tabulated in [16].

\begin{tabular}{llllll}
\hline & \multicolumn{5}{c}{ Branching fractions (\%) } \\
\cline { 2 - 6 } Transition & SE & Tayal $^{\text {a }}$ & Froese Fischer $^{\mathrm{b}}$ & Hibbert $^{\mathrm{c}}$ & Measured ${ }^{\mathrm{d}}$ \\
\hline${ }^{3} \mathrm{P}_{0}^{\prime} \leftarrow{ }^{3} \mathrm{P}_{1}^{\mathrm{o}^{\prime}}$ & 33.1 & 32.9 & 33.3 & 33.4 & $35.9(2.7)$ \\
${ }^{3} \mathrm{P}_{1} \leftarrow$ & 24.5 & 24.3 & 24.6 & 24.8 & $25.4(1.4)$ \\
${ }^{3} \mathrm{P}_{2}^{\prime} \leftarrow$ & 41.0 & 40.9 & 41.3 & 41.8 & $38.7(2.6)$ \\
${ }^{1} \mathrm{D}_{2}^{\prime} \leftarrow$ & 1.3 & 2.0 & 0.8 & - & - \\
${ }^{1} \mathrm{~S}_{0}^{\prime} \leftarrow$ & 0.12 & - & 0.04 & - & - \\
${ }^{3} \mathrm{P}_{1} \leftarrow{ }^{3} \mathrm{P}_{2}^{\mathrm{o}}$ & 25.2 & 25.3 & 25.3 & 25.3 & $26.7(1.6)$ \\
${ }^{3} \mathrm{P}_{2}^{\prime} \leftarrow$ & 74.8 & 74.7 & 74.7 & 74.7 & $73.3(3.3)$ \\
${ }^{1} \mathrm{D}_{2}^{\prime} \leftarrow$ & 0.005 & 0.0003 & 0.025 & - & - \\
& & & & & \\
${ }^{3} \mathrm{P}_{0}^{\prime} \leftarrow{ }^{1} \mathrm{P}_{1}^{\mathrm{o}^{\prime}}$ & 0.22 & 5.2 & 0.3 & - & - \\
${ }^{3} \mathrm{P}_{1} \leftarrow$ & 0.25 & 4.4 & 0.22 & - & - \\
${ }^{3} \mathrm{P}_{2}^{\prime} \leftarrow$ & 0.11 & 4.0 & 0.16 & - & - \\
${ }^{1} \mathrm{D}_{2}^{\prime} \leftarrow$ & 88.8 & 81.1 & 93.2 & - & - \\
${ }^{1} \mathrm{~S}_{0}^{\prime} \leftarrow$ & 10.6 & 5.3 & 6.1 &
\end{tabular}

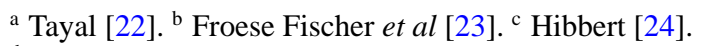

${ }^{\mathrm{d}}$ Federman et al [21].

Lifetimes have been measured for the upper levels with $J=2,1$ and 0 [21]. The results were: $\tau\left({ }^{3} \mathrm{P}_{2}\right)=$ $0.84 \pm 0.07 ; \tau\left({ }^{3} \mathrm{P}_{1}\right)=0.79 \pm 0.06 ;$ and $\tau\left({ }^{3} \mathrm{P}_{0}\right)=0.79 \pm 0.10$. The predicted ratios of these lifetimes, obtained from the wavelength and mixing angle data [16] and equation (12) are

$$
\tau\left({ }^{3} \mathrm{P}_{2}\right) / \tau\left({ }^{3} \mathrm{P}_{1}\right)=1.0004 ; \quad \tau\left({ }^{3} \mathrm{P}_{1}\right) / \tau\left({ }^{3} \mathrm{P}_{0}\right)=1.0098,
$$

whereas the measured ratios are

$\tau\left({ }^{3} \mathrm{P}_{2}\right) / \tau\left({ }^{3} \mathrm{P}_{1}\right)=1.06 \pm 0.12 ; \quad \tau\left({ }^{3} \mathrm{P}_{1}\right) / \tau\left({ }^{3} \mathrm{P}_{0}\right)=1.00 \pm 0.15$

showing that the consistency applies here as well.
5.3. Predicted branching fractions for the $\mathrm{Si}, \mathrm{Ge}$, and $\mathrm{Sn}$ isoelectronic sequences

The success of this approach for branching fractions in the neutral atoms Si I, Ge I, Sn I and the singly-ionized atom P II provides confidence that similar accuracy can be attained by extending the semiempirical calculations to multiplycharged ions in these isoelectronic sequences. Wavelengths and branching fractions for the homologous $n \mathrm{~s}^{2} n \mathrm{p}^{2}-n \mathrm{~s}^{2} n$ $\mathrm{p}(n+1) \mathrm{s}$ transitions have been reported for P II-Cl IV in the $\mathrm{Si}(n=3)$ sequence [16], As II-Br IV in the Ge $(n=4)$ sequence [16], and for Sb III-Cs VI in the Sn $(n=5)$ sequence [17].

The results for the Sn sequence are indicative of the ability of the method to isoelectronically span a wide range 
Table 3. Wavelengths and semiempirical branching fractions (in \%) for the $5 s^{2} 5 p^{2}-5 s^{2} 5 p 6 s$ transitions for ions of the Sn sequence. The energy level database is tabulated in [17].

\begin{tabular}{|c|c|c|c|c|c|c|c|c|c|c|}
\hline \multirow[b]{2}{*}{ Transition } & \multicolumn{2}{|c|}{$\mathrm{Sb}$ II } & \multicolumn{2}{|c|}{ Te III } & \multicolumn{2}{|c|}{ I IV } & \multicolumn{2}{|c|}{$\mathrm{Xe} \mathrm{V}$} & \multicolumn{2}{|c|}{ Cs VI } \\
\hline & $\lambda(\AA)$ & $B_{\mathrm{ul}}$ & $\lambda(\AA)$ & $B_{\mathrm{ul}}$ & $\lambda(\AA)$ & $B_{\mathrm{ul}}$ & $\lambda(\AA)$ & $B_{\mathrm{ul}}$ & $\lambda(\AA)$ & $B_{\mathrm{ul}}$ \\
\hline${ }^{3} \mathrm{P}_{0}^{\prime} \leftarrow{ }^{3} \mathrm{P}_{1}^{\mathrm{o}^{\prime}}$ & 1438.1 & 30.2 & 928.3 & 30.1 & 666.3 & 30.0 & 512.8 & 31.1 & 430.3 & 31.0 \\
\hline${ }^{3} \mathrm{P}_{1} \leftarrow$ & 1504.2 & 16.1 & 971.2 & 15.6 & 698.0 & 15.3 & 538.6 & 16.1 & 431.9 & 15.6 \\
\hline${ }^{3} \mathrm{P}_{2}^{\prime} \leftarrow$ & 1565.5 & 43.4 & 1004.4 & 45.1 & 718.9 & 49.0 & 552.9 & 50.0 & 442.3 & 51.2 \\
\hline${ }^{1} \mathrm{D}_{2}^{\prime} \leftarrow$ & 1762.2 & 9.5 & 1106.6 & 8.5 & 784.0 & 5.0 & 600.4 & 2.4 & 479.3 & 1.8 \\
\hline${ }^{3} \mathrm{~S}_{0}^{\prime} \leftarrow$ & 2190.9 & 0.8 & 1310.6 & 0.7 & 885.7 & 0.7 & 664.8 & 0.4 & 522.7 & 0.3 \\
\hline${ }^{3} \mathrm{P}_{1} \leftarrow$ & 84.7 & & 13.6 & & 649.3 & 27 & 500.6 & 27. & 402.0 & 27.7 \\
\hline${ }^{3} \mathrm{P}_{2}^{\prime} \leftarrow$ & 1436.5 & 65.5 & 943.0 & 63.6 & 667.3 & 57.8 & 513.0 & 54.3 & 411.0 & 51.1 \\
\hline${ }^{1} \mathrm{D}_{2}^{\prime} \leftarrow$ & 1600.4 & 6.6 & 1032.5 & 8.9 & 723.0 & 14.6 & 553.5 & 18.1 & 442.7 & 21.2 \\
\hline${ }^{3} \mathrm{P}_{0}^{\prime} \leftarrow{ }^{1} \mathrm{P}_{1}^{\mathrm{o}^{\prime}}$ & 1317.5 & 3.0 & 866.4 & 2.1 & 619.7 & 1. & 469.2 & 0. & 378.5 & 0.6 \\
\hline${ }^{3} \mathrm{P}_{1} \leftarrow$ & 1372.8 & 6.0 & 903.6 & 5.6 & 647.0 & 5.2 & 490.6 & 4.3 & 396.8 & 4.4 \\
\hline${ }^{3} \mathrm{P}_{2}^{\prime} \leftarrow$ & 1423.7 & 0.6 & 932.4 & 1.4 & 664.9 & 5.2 & 502.6 & 9.4 & 405.5 & 11.9 \\
\hline${ }^{1} \mathrm{D}_{2}^{\prime} \leftarrow$ & 1584.6 & 80.2 & 1019.8 & 79.2 & 720.2 & 74.8 & 541.4 & 71.6 & 436.4 & 68.6 \\
\hline${ }^{1} \mathrm{~S}_{0}^{\prime} \leftarrow$ & 1923.3 & 10.2 & 1190.5 & 11.7 & 805.1 & 13.3 & 593.3 & 14.1 & 472.1 & 14.6 \\
\hline
\end{tabular}

of wavelengths, and are reproduced here in table 3 . The applicability of the method is limited only by the availability of measured energy level data. Thus, by appropriate choice of ion in table 3 , intensity standards from 2000 to $400 \AA$ are directly available.

\section{Theoretical calculation of branching fractions}

$A b$ initio theoretical calculations also provide a possible source of branching fractions. However, for heavy and highly ionized systems, many uncertainties presently exist concerning the accuracy and reliability of purely theoretical methods. In heavy complex ions, theoretical values for lifetimes often differ significantly from measured lifetimes (e.g. [25]). The origin of these discrepancies is difficult to determine, since the reciprocal lifetime represents a sum over a manifold of theoretical transition probabilities. Since no database currently exists for branching fractions in these systems, theory is untested in this regime.

Moreover, it often happens that the inclusion of additional configurations in a multiconfiguration calculation not only causes significant changes in the values for the predicted energy values, but also changes the ordering of the levels. Thus, without experimental knowledge, it is not even possible to prescribe the exit channels that are energetically allowed for a given upper level.

Even in cases where theoretical calculations agree with experimental measurements and with semiempirical estimates for transitions that are spin-allowed in $L S$ coupling, various calculations differ greatly from each other for the transitions that are spin-forbidden in $L S$ coupling (cf table 2). For these spin-forbidden transitions, the semiempirical calculations (which use measured energy levels) may be more reliable than $a b$ initio estimates (that may predict energies that differ significantly from experimental values).

This underscores the need for experimental measurements of branching fractions in heavy, highly ionized atoms. Comparison of $a b$ initio calculations with an experimental database could indicate whether relative values obtained for branching fractions are more reliable than the absolute values summed to obtain lifetimes. This would also permit comparisons with experiment for the various types of $a b$ initio methods (e.g. multiconfiguration Dirac-Fock, relativistic random phase approximation, many-body perturbation theory, etc).

\section{Relativistic corrections: the $\mathrm{Pb}$ sequence}

The semiempirical methods discussed above were modelled on the assumptions of the nonrelativistic Schrödinger equation, which uses an $L S$-coupling angular basis set and radial wavefunctions that are independent of $J$. These methods have been shown to be applicable to systems of low to moderate nuclear charge $Z$, but for high- $Z$ atoms relativistic corrections can become significant. In order to treat such cases by the semiempirical methods described above, the formulation has been extended to a model based on the relativistic Dirac equation, which uses a $j j$-coupling basis set and $j$-dependent radial wavefunctions. This formulation involves two single particle $j$-dependent radial matrix elements, $R_{11}=\left\langle s_{1 / 2}|r| p_{1 / 2}\right\rangle$ and $R_{13}=\left\langle s_{1 / 2}|r| p_{3 / 2}\right\rangle$. The nonrelativistic results are recovered when the ratio $R_{13} / R_{11}$ is close to unity. Simple algebraic expressions are also available for these relativistic angular factors $[3,26]$.

In order to investigate the significance of these relativistic corrections, $a b$ initio theoretical calculations have been carried out [26] for the quantity $R_{13} / R_{11}$ for the transitions $n \mathrm{~s}^{2} n \mathrm{p}^{2}-n \mathrm{~s}^{2} n \mathrm{p}(n+1) \mathrm{s}$ for the Si $(n=3)$, Ge $(n=4)$, $\mathrm{Sn}(n=5)$ and $\mathrm{Pb}(n=6)$ sequences using the multiconfiguration Dirac-Fock program GRASP [27]. The calculations indicated that the ratio $R_{13} / R_{11}$ is very close to unity for the $\mathrm{Si}, \mathrm{Ge}$, and $\mathrm{Sn}$ sequences, and relativistic corrections for these ions were insignificant. However, for the $\mathrm{Pb}$ sequence the ratios differed substantially from unity (1.4590 and 1.4224 for $\mathrm{Pb} \mathrm{I}$ and $\mathrm{Bi} \mathrm{II})$, and relativistic corrections were necessary.

Table 4 shows these calculations for the first two members of the $\mathrm{Pb}$ sequence (higher members of this sequence are radioactive). Since the ${ }^{3} \mathrm{P}_{2}^{\mathrm{o}}$ and ${ }^{1} \mathrm{P}_{1}^{\mathrm{o}^{\prime}}$ levels lie 
Table 4. Relativistic corrections to branching fractions for the ${ }^{3} \mathrm{P}_{1}^{\mathrm{o}}$ upper level in the $6 \mathrm{~s}^{2} 6 \mathrm{p}^{2}-6 \mathrm{~s}^{2} 6 \mathrm{p} 7 \mathrm{~s}$ multiplet in $\mathrm{Pb}$ I and Bi II.

\begin{tabular}{lllllll}
\hline & \multicolumn{3}{c}{$\mathrm{Pb} \mathrm{I}$} & & \multicolumn{2}{c}{ Bi II } \\
\cline { 2 - 4 } Transition & $B_{\mathrm{ul}}(\mathrm{N})^{\mathrm{a}}$ & $B_{\mathrm{ul}}(\mathrm{R})^{\mathrm{b}}$ & $B_{\mathrm{ul}}(\mathrm{M})^{\mathrm{c}}$ & & $B_{\mathrm{ul}}(\mathrm{N})^{\mathrm{a}}$ & $B_{\mathrm{ul}}(\mathrm{R})^{\mathrm{d}}$ \\
\hline${ }^{3} \mathrm{P}_{0}^{\prime} \leftarrow{ }^{3} \mathrm{P}_{1}^{\mathrm{o}}{ }^{\prime}$ & 0.489 & 0.310 & 0.324 & 0.43 & 0.25 \\
${ }^{3} \mathrm{P}_{1} \leftarrow$ & 0.128 & 0.166 & 0.188 & 0.12 & 0.16 \\
${ }^{3} \mathrm{P}_{2}^{\prime} \leftarrow$ & 0.381 & 0.520 & 0.500 & 0.44 & 0.59 \\
${ }^{1} \mathrm{D}_{2}^{\prime} \leftarrow$ & 0.0029 & 0.0040 & 0.0005 & 0.004 & 0.005 \\
${ }^{1} \mathrm{~S}_{0}^{\prime} \leftarrow$ & $7 \times 10^{-5}$ & $3 \times 10^{-5}$ & - & 0.0002 & 0.0009 \\
\hline
\end{tabular}

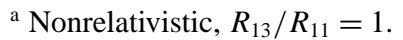

${ }^{\mathrm{b}}$ Relativistic, $R_{13} / R_{11}=1.4590$.

${ }^{c}$ Measured, [28].

${ }^{\mathrm{d}}$ Relativistic, $R_{13} / R_{11}=1.4224$.

much higher in energy than ${ }^{3} \mathrm{P}_{1}^{\mathrm{o}}$ and have additional decay channels to other configurations, only transitions from the ${ }^{3} \mathrm{P}_{1}^{\mathrm{o}^{\prime}}$ upper level are listed. For the neutral atom $\mathrm{Pb} \mathrm{I}$, branching fraction measurements are available [28]. These show that while the relativistic corrections are substantial, the use of the relativistic formulation yields values in agreement with measured branching fractions. These methods were then used to calculate the branching fractions in $\mathrm{Bi}$ II, also presented in table 4.

\section{Summary}

The existing atomic database possesses a serious deficiency in measured values for emission transition probabilities and absorption oscillator strengths for branched transitions in multiply-charged ions. To remedy this situation, existing lifetime measurements must be complemented by branching fraction measurements. Such measurements are hindered by the lack of line intensity calibration standards in the VUV. If atomic spectroscopy is to fulfil its role as an enabling science, priority should be given to correcting this deficiency. A concerted effort is needed to produce a set of conveniently applied and universally accepted measured line intensity standards. In the meantime, transitions exist in certain ions that have been shown to be virtually free of CI, and thus have relative intensities that can be accurately specified from IC amplitudes deduced from observed energy level data.

\section{Acknowledgments}

The work was supported by NASA grants NAG5-11440 and NNG06GC70G. ST acknowledges support from NSF grant No. 0353899.

\section{References}

[1] Curtis L J 2005 Precision oscillator strengths and lifetime measurements Springer Handbook of Atomic Molecular and Optical Physics ed G W F Drake (Berlin: Springer) Chapter 17 259-66

[2] Martin W C and Wiese W L 2005 Atomic spectroscopy Springer Handbook of Atomic Molecular and Optical Physics ed G W F Drake (Berlin: Springer) Chapter 10 175-98

[3] Curtis L J 2003 Atomic Structure and Lifetimes: A Conceptual Approach (Cambridge: Cambridge University Press)

[4] Curtis L J and Martinson I 1999 Lifetimes of excited states in highly charged ions Atomic Physics with Heavy Ions ed H F Beyer and V P Shevelko (Berlin: Springer) 197-218

[5] Adams D L and Whaling W 1981 J. Opt. Soc. Am 711036

[6] Danzmann S and Kock M 1982 J. Opt. Soc. Am. 721556

[7] Hashiguchi I and Hasikuni M 1985 J. Phys. Soc. Japan 541290

[8] Whaling W, Carle M T and Pitt M L 1993 J. Quant. Spectrosc. Radiat. Transfer $\mathbf{5 0} 7$

[9] Bridges J M and Ott W R 1977 Appl. Opt. 16367

[10] Saunders R D, Ott W R and Bridges J M 1978 Appl. Opt. 17593

[11] Lawler J E, Bergeson S D, Fedchak J A and Mullman K L 2005 Phys. Scr. T 8311

[12] Danzmann K, Günther M, Fischer J, Kock M and Kühne M 1988 Appl. Opt. 274947

[13] Heise C, Hollandt J, Kling R, Kock M and Kühn M 1994 Appl. Opt. 225111

[14] Nave G, Li Z, Sansonetti C J, Griesmann U and Fried A D 2005 Phys. Scr. T 11935

[15] Curtis L J 1998 J. Phys. B: At. Mol. Opt. Phys. 31 L769

[16] Curtis L J 2000 J. Phys. B: At. Mol. Opt. Phys. 33 L259

[17] Curtis L J 2001 Phys. Scr. 63104

[18] Smith P L, Huber M C E, Tozzi G P, Greisinger H H, Cardon B L and Lombardi G G 1987 Astrophys. J. 322573

[19] Li Z-S, Norin J, Persson A, Wahlström C-G, Svanberg S, Doidge P S and Biémont E 1999 Phys. Rev. A 60198

[20] Lotrian J, Guern Y, Cariou J and Johannin-Gilles A 1977 J. Quant. Spectrosc. Radiat. Transfer 18509

[21] Federman S R, Brown M, Torok S, Cheng S, Irving R E, Schectman R M and Curtis L J 2006 Astrophys. J. at press

[22] Tayal S S 2003 Astrophys. J., Suppl. Ser. 146459

[23] Froese Fischer C, Tachiev G and Irimia A 2006 At. Data Nucl. Data Tables 92607

[24] Hibbert A 1988 Phys. Scr. 3837

[25] Beideck D J, Curtis L J, Irving R E, Maniak S T, Hellborg R, Johansson S G, Martinson I and Rosberg M 1993 J. Opt. Soc. Am. B 10977

[26] Curtis L J, Ellis D G, Matulioniene R and Brage T 1997 Phys. Scr. 56240

[27] Parpia F A, Fischer C F and Grant I P 1996 Comp. Phys. Commun. 94249

[28] Lotrian J, Guern Y, Cariou J and Johannin-Gilles A 1979 J. Quant. Spectrosc. Radiat. Transfer 21143 